\title{
Metallomics
}

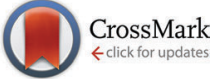

Cite this: Metallomics, 2016, 8, 305

Received 28th October 2015, Accepted 6th January 2016

DOI: $10.1039 / c 5 m t 00279 f$

www.rsc.org/metallomics

\section{Distribution of selenium in zebrafish larvae after exposure to organic and inorganic selenium forms $\dagger$}

\author{
N. V. Dolgova, ${ }^{a}$ M. J. Hackett, ${ }^{a}$ T. C. MacDonald, ${ }^{a b c}$ S. Nehzati, ${ }^{a}$ A. K. James, ${ }^{a b c}$ \\ P. H. Krone, ${ }^{b c}$ G. N. George ${ }^{\star a b d}$ and I. J. Pickering*abd
}

\begin{abstract}
Selenium is an essential micronutrient for many organisms, and in vertebrates has a variety of roles associated with protection from reactive oxygen species. Over the past two decades there have been conflicting reports upon human health benefits and detriments arising from consumption of selenium dietary supplements. Thus, early studies report a decrease in the incidence of certain types of cancer, whereas subsequent studies did not observe any anti-cancer effect, and adverse effects such as increased risks for type 2 diabetes have been reported. A possible contributing factor may be that different chemical forms of selenium were used in different studies. Using larval stage zebrafish (Danio rerio) as a model organism, we report a comparison of the toxicities and tissue selenium distributions of four different chemical forms of selenium. We find that the organic forms of selenium tested (Se-methylL-selenocysteine and L-selenomethionine) show considerably more toxicity than inorganic forms (selenite and selenate), and that this appears to be correlated with the level of bioaccumulation. Despite differences in concentrations, the tissue specific pattern of selenium accumulation was similar for the chemical forms tested; selenium was found to be highly concentrated in pigment (melanin) containing tissues especially for the organic selenium treatments, with lower concentrations in eye lens, yolk sac and heart. These results suggest that pigmented tissues might serve as a storage reservoir for selenium.
\end{abstract}

\section{Introduction}

Selenium is an essential micronutrient for organisms spanning all three domains of life, ranging from archaea to humans. In vivo, most functional selenium exists as L-selenocysteine, the twenty first amino acid. L-Selenocysteine is an essential part of the catalytic centre of several enzymes functioning to protect aerobic organisms against oxidative stress and in thyroid hormonal regulation. ${ }^{1}$ Selenium also occurs in vivo as L-selenomethionine, which has no known functional roles and is likely present because sulfur and selenium have similar chemistries, and L-selenomethionine and L-methionine are difficult to distinguish

\footnotetext{
${ }^{a}$ Molecular and Environmental Science Research Group, Department of Geological Sciences, University of Saskatchewan, Saskatoon, SK, S7N 5E2, Canada.

E-mail: ingrid.pickering@usask.ca,g.george@usask.ca

${ }^{b}$ Toxicology Centre, University of Saskatchewan, Saskatoon, SK, S7N 5B3, Canada

${ }^{c}$ Department of Anatomy and Cell Biology, University of Saskatchewan, Saskatoon, SK, S7N 5E5, Canada

${ }^{d}$ Department of Chemistry, University of Saskatchewan, Saskatoon, SK, S7N 5C9, Canada

$\dagger$ Electronic supplementary information (ESI) available: Fig. S1-S5 showing fish larval deformities and deaths as a function of selenium species, concentration and exposure time, and selenium correlation with iron. See DOI: 10.1039/c5mt00279f
}

biochemically. L-Selenomethionine is toxic to vertebrates through unknown mechanisms.

In the past two decades, consumption of selenium supplements has become widespread, but whether or not these are beneficial depends on a number of factors, such as the chemical form of selenium being consumed and the selenium status of the individual. Thus, while some reports suggest that selenium supplements can decrease the incidence of some types of cancer, ${ }^{2}$ others show no effect of selenium supplements on cancer, and some correlate selenium supplement consumption with adverse effects such as substantially increased incidence of type 2 diabetes. ${ }^{3,4}$ In many of these studies both the selenium status of the cohort individuals and the chemical forms of selenium are either poorly defined or unknown. We hypothesize that apparently conflicting information about the benefits of selenium may in part be due to different selenium chemical forms in the various supplements used.

Dietary selenium can include several forms which can be divided into organic and inorganic. Organic forms might include L-selenomethionine, Se-methyl-L-selenocysteine and L-selenocysteine derivatives. Inorganic forms might be selenite, selenate or elemental selenium, which itself could be present as either bio-available nano-particulate forms, or bio-unavailable amorphous or crystalline selenium. All of these forms have significantly different 
absorption efficiencies, metabolism pathways and toxicity profiles. $^{5}$ While it is important to understand the differences in tissue distribution and metabolism of different selenium forms, at present information on these differences is very limited.

Zebrafish (Danio rerio) is increasingly used as a model organism in biochemical, physiological and toxicological studies. ${ }^{6,7}$ Many genetic pathways involved in vertebrate development, cancer, diabetes and other conditions are evolutionally conserved and very similar to the pathways in mammals including humans. Short developmental span, relative easiness of genetic modifications and transparency of the larvae make zebrafish an attractive model organism for toxicological research.

The importance of environmental selenium is also increasingly acknowledged. Agricultural irrigation and industrial activities such as wastewater discharges from mining, coal power plants and oil refining, lead to selenium enrichment of soil and water bodies. In water systems increasing selenium concentrations only 7-30 times over required nutritional levels can result in adverse effects in top predators. ${ }^{8}$ Zebrafish are already an established model for evaluation of the toxicological impact of selenium compounds, ${ }^{9-12}$ and our previous work found that endogenic selenium is concentrated in pigment-containing cells of zebrafish larvae, ${ }^{13}$ and that zebrafish embryos from L-selenomethionine-treated mothers have elevated Se in the eye lens. ${ }^{14}$ Here we extend these studies to examine the toxicity and tissue distribution of four different chemical forms of selenium administered in fish culture water.

\section{Experimental}

\section{Toxicological profiles}

All procedures involving zebrafish were approved by the University of Saskatchewan Ethics Board. Embryos were collected within 2 hours post fertilization (hpf), rinsed with system water and placed into Petri dishes with $0.5 \times$ embryo media $(7.5 \mathrm{mM} \mathrm{NaCl}$, $0.25 \mathrm{mM} \mathrm{KCl}, 0.5 \mathrm{mM} \mathrm{MgSO}{ }_{4}, 75 \mu \mathrm{M} \mathrm{KH} \mathrm{PO}_{4}, 25 \mu \mathrm{M} \mathrm{Na}_{2} \mathrm{HPO}_{4}$, $0.5 \mathrm{mM} \mathrm{CaCl}_{2}, 0.35 \mathrm{mM} \mathrm{NaHCO}_{3}, \mathrm{pH} \sim 7.0$ ) with no more than 50 embryos per dish. Every 24 hours the embryo media in each dish was exchanged for a fresh aliquot, and dead embryos and empty egg chorions left after hatching were removed. Embryos were grown in an incubator at $28{ }^{\circ} \mathrm{C}$. For toxicity studies, zebrafish were divided into groups of 25 larvae per Petri dish with three or four groups being allocated for each selenium concentration. The volume of $0.5 \times$ embryo media was kept at $24 \mathrm{ml}$, and $1 \mathrm{ml}$ of $25 \times$ solution of a selenium compound was added when fish reached $72 \mathrm{hpf}$. Stock solutions of selenium compounds were prepared in deionized water (Millipore) using sodium selenate (anhydrous, Alfa Aesar), sodium selenite (anhydrous, Alfa Aesar), L-selenomethionine (Chem-Implex Int'l Inc., USA) and Se-methyl-L-selenocysteine hydrochloride (Sigma). For the duration of the study, all stock solutions were kept frozen at $-20{ }^{\circ} \mathrm{C}$ and thawed right before use. The $0.5 \times$ embryo media with selenium compound was changed every 24 hours, and at the same time larvae were examined for deformities and lethalities. Treatment continued until 7 days post fertilization (dpf), after which larvae were euthanized with $0.2 \%$ Tricaine and disposed of following procedures established by the Laboratory Animal Service Unit of University of Saskatchewan.

\section{$\mathrm{X}$-ray fluorescence imaging (XFI) sample preparation}

The X-ray fluorescence imaging (XFI) ${ }^{15}$ study of selenium in zebrafish larvae followed the design used for the study of mercury distribution conducted previously in our research group ${ }^{16-19}$ with some modifications. Because of the high water solubility of some selenium compounds and in order to preserve the selenium distribution in tissues, we employed cryosections ${ }^{20}$ rather than the previously used methacrylate-embedded sections. After being grown as described above to $72 \mathrm{hpf}$, larvae were divided into groups of 25 animals per Petri dish and treated with $1 \mu \mathrm{M}$ selenomethionine, $1 \mu \mathrm{M}$ Se-methyl-selenocysteine, $200 \mu \mathrm{M}$ selenite, $200 \mu \mathrm{M}$ selenate (final concentrations) or control (no selenium) for 24 hours. Following treatment, fish were euthanized with Tricaine, placed into cryomolds with optimal cutting temperature (OCT) media (Tissue-Tek) and flash frozen in liquid-nitrogen chilled liquid isopentane. Frozen blocks were stored at $-80{ }^{\circ} \mathrm{C}$ until sectioning. $10 \mu \mathrm{m}$-thick cryosections were prepared using a ESBE Scientific (Markham, ON, Canada) HM550 cryostat microtome. Sections were transferred to Thermanox plastic slides for XFI and to Fisherbrand Superfrost Plus microscope slides for histological staining. After XFI samples were also stained with haemotoxylin and eosin (H\&E).

\section{$\mathrm{X}$-ray fluorescence imaging and data analysis}

$\mathrm{X}$-ray fluorescence imaging ${ }^{15}$ was performed at the Stanford Synchrotron Radiation Lightsource (SSRL) with the storage ring operating at $500 \mathrm{~mA}$ and $3 \mathrm{GeV}$ using beamline 2-3. Samples were mounted at a $45^{\circ}$ angle to the incident X-ray beam and raster-scanned with a step size of $5 \mu \mathrm{m}$ and a dwell time of $600 \mathrm{~ms}$ at the incident energy of $13450 \mathrm{eV}$. X-ray fluorescence was monitored using a silicon-drift Vortex detector (Hitachi High Technologies Science America Inc., Northridge, CA, USA). Data reduction and analysis were carried out as previously described. ${ }^{13-19}$ Elemental concentrations were quantified using fluorescence intensities from thin standard foils (Micromatter, Vancouver, $\mathrm{BC}$, Canada) recorded under the same conditions as the larval sections. Analysis of correlations between selenium and zinc was performed as described previously. ${ }^{13}$ Values for $R^{2}$ (the coefficient of determination) were computed with exclusion of points having concentrations less than $10 \%$ of the maximum in order to eliminate uncorrelated baseline points, as previously described. ${ }^{13}$

\section{Results and discussion}

\section{Toxicological profiles}

Larvae at $3 \mathrm{dpf}$ were exposed to selenite, selenate, L-selenomethionine or Se-methyl-L-selenocysteine by adding solutions of different concentration to embryo media. The full results of these experiments are presented in ESI $\dagger$ (Fig. S1-S4) showing observed fish deformities and deaths as a function of selenium species, concentration and treatment time. We observed several 
types of deformities in the larvae, including scoliosis, kyphosis, lordosis and edema, all of which previously have been described in zebrafish larvae exposed to L-selenomethionine via maternal transfer. ${ }^{10}$ Using three to four groups of 25 fish larvae (total 75-100 zebrafish) for each concentration, we find that the two organic forms of selenium are much more toxic for zebrafish larvae at this developmental stage than are the two inorganic forms tested. Thus, concentrations above $1 \mu \mathrm{M}$ of L-selenomethionine or Se-methyl-L-selenocysteine are lethal (Fig. S1 and S2, ESI $\dagger$ ), whereas aqueous selenite and selenate show comparable effects only above $1 \mathrm{mM}$. We also find that Se-methyl-L-selenocysteine is more toxic than L-selenomethionine, and that selenite $\left[\mathrm{SeO}_{3}\right]^{2-}$ is more toxic than selenate $\left[\mathrm{SeO}_{4}\right]^{2-}$.

Our results that organic selenium forms are more toxic than inorganic forms confirm the trends observed by Niimi and $\mathrm{LaHam}^{21}$ for zebrafish larvae exposed at the somewhat earlier developmental stage of 12-36 hpf, for which selenite was observed to be more toxic than selenate, and L-selenomethionine was the most toxic of all tested species. Our observations are also consistent with the recent study of Arnold et al. ${ }^{22}$ using zebrafish embryos at the 8-32 cell stage (approximately $2 \mathrm{hpf}$ ) that L-selenomethionine caused significant deformities at a level of $100 \mu \mathrm{g} \mathrm{L}^{-1}$ relative to control whereas selenite and selenite did not. In other studies, it has been reported that selenomethionine is more toxic compared to selenite in rats; ${ }^{23}$ apparently in contradiction of this, others have reported that inorganic selenium compounds are more toxic to rats than selenomethionine, though the trends were not marked. ${ }^{24}$ Variations between studies might be attributable to differences in administration of the selenium forms, in excretion of selenium and in gastrointestinal microflora, ${ }^{25}$ which may modify the selenium form before absorption. Such variations do not apply to our current zebrafish study.

Approximately 24 hours prior to the appearance of deformities or lethalities in our experiments, we observed that larvae typically became immobile and appeared moribund. At this stage, microscopic examination of larvae showed that the circulating blood (seen best in the heart) had exhibited a color change from the typical red-pink observed with healthy larvae to a greyish-brown with a loss of color that increased with time. This effect was seen for all four selenium species tested, although at substantially lower concentration with organic forms when compared with inorganic forms.

\section{XFI of untreated zebrafish larvae}

Since selenoproteins are expressed in all tissues and organs of vertebrates, endogenous selenium is expected to be found everywhere in the body. Selenium in control larvae, with no administered selenium, arises largely from maternal transfer during vitellogenesis, or from traces of selenium in the embryo media. We used X-ray fluorescence imaging (XFI) to investigate the tissue dependence of selenium accumulation. The results of XFI experiments on larval eye, brain and trunk sections are shown in Fig. 1-3, respectively. The eye section data (Fig. 1) show elevated levels of selenium in the eye lens and in the melanocyte-containing layers of the eye; the retinal pigmented epithelium (RPE) and choroidal melanocytes. At the early

developmental stage of zebrafish that was used for XFI (96 hpf) RPE and choroid melanocytes are hard to distinguish, even on histologically stained specimens, and in any case the XFI resolution used cannot spatially resolve these two layers.

For the XFI data presented herein, measured on SSRL 2-3, the endogenous selenium in control fish is below the limits of detection. In previous work using 20-ID at the Advanced Photon Source (APS) low levels of endogenous selenium were observed to be preferentially accumulated in pigmented regions (melanincontaining tissues) throughout the larval zebrafish body. ${ }^{13}$ This difference is directly attributable to the much greater X-ray photon flux on APS 20-ID compared to SSRL 2-3, rather than any physical difference in the samples. However, the levels of zinc are substantially higher than those of selenium, ${ }^{13,17}$ and the zinc distribution is thus very easily detected in our data (Fig. 1-3). Our XFI of cryosections confirm our previous findings on fixed embedded sections ${ }^{13,17}$ that melanocyte-rich tissues contain high levels of zinc, in both control and selenium-treated larvae (Fig. 1-3). Our results also confirm previous findings that the RPE and choroid melanocytes of the eye are rich in zinc. ${ }^{26,27}$ As previously discussed, ${ }^{13,17}$ high levels of zinc are likely to be the result of metal ion binding to melanin, with some contribution from zinc-containing metalloproteins such as metallothioneins that are integral to the RPE and choroid melanocytes.

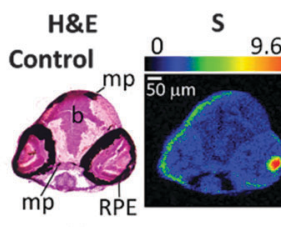

Selenomethionine
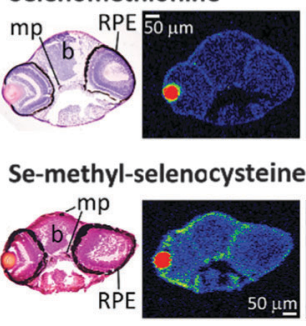

Selenate

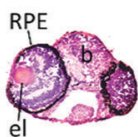

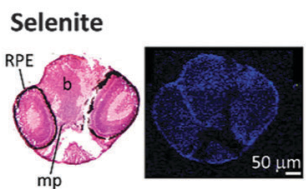
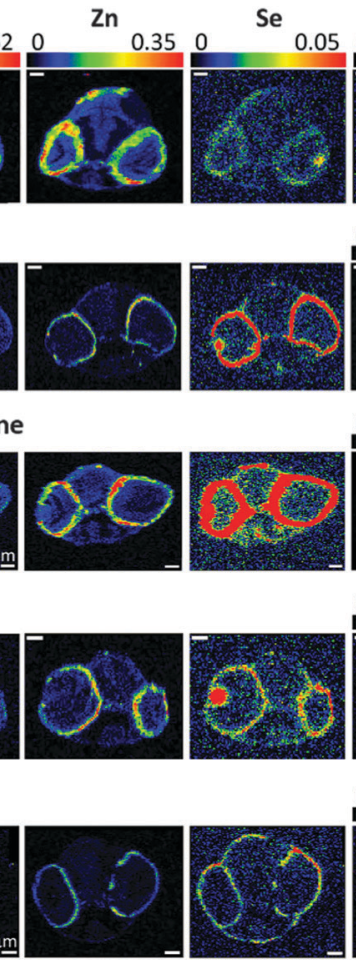

Fig. 1 XFI of eye sections of larval zebrafish treated with different forms of selenium. Each row corresponds to a different treatment, as indicated, with the corresponding H\&E stained optical micrograph shown adjacent. The three left XFI columns show the XFI of S, Zn and Se, respectively, with units of areal density (in $\mu \mathrm{g} \mathrm{cm}^{-2}$ ) on a common scale for each element. The Se XFI in the right column shows XFI with independent intensity scales $\left(\mu \mathrm{g} \mathrm{cm}^{-2}\right.$ ). Scale bars indicate $50 \mu \mathrm{m}$. Abbreviations: RPE - retinal pigmented epithelium, mp - melanophores, el - eye lens, b - brain. 

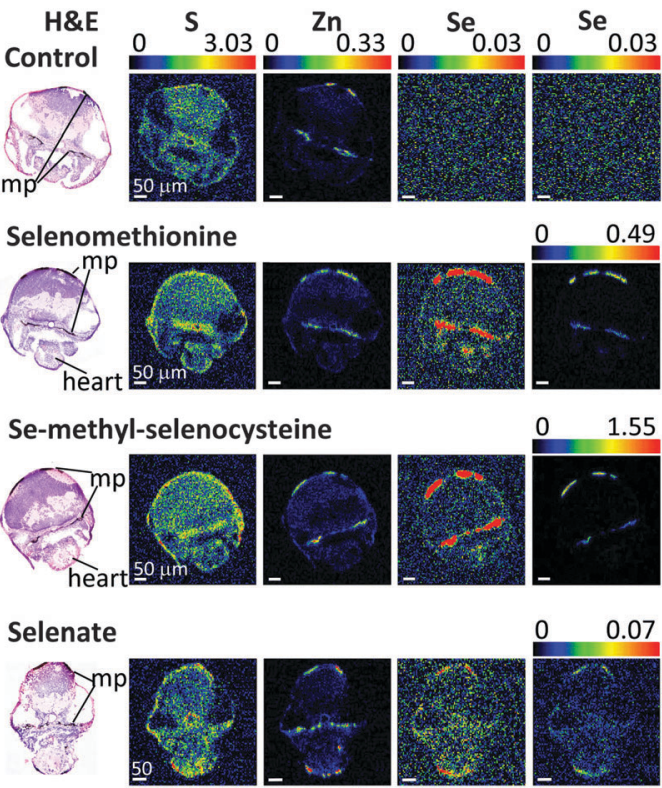

Selenite
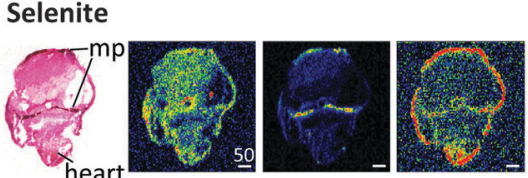

$0 \quad 0.10$

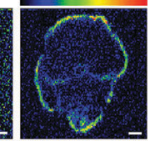

Fig. $2 \mathrm{XFI}$ of brain sections of larval zebrafish treated with different forms of selenium. Details are similar to those in Fig. 1. Scale bars indicate $50 \mu \mathrm{m}$. Abbreviations: $\mathrm{mp}$ - melanophores

\section{XFI of selenium-treated zebrafish larvae}

Zebrafish larvae for XFI measurements (72 hpf) were treated with four different selenium compounds for 24 hours, with concentrations chosen from the toxicological profiles (Fig. S1-S4, ESI $\dagger$ ) to be as high as possible without causing death or deformity. As described above, since the organic selenium compounds were much more toxic relative to inorganic forms, fish were treated with $200 \mu \mathrm{M}$ of selenite or selenate or with $1 \mu \mathrm{M}$ of L-selenomethionine or Se-methylL-selenocysteine. Despite this 200-fold lower exposure concentration, the selenium levels were observed to be higher in fish treated with organic forms (Fig. 1-3). Moreover, of the two organic forms tested, the more toxic Se-methyl-L-selenocysteine accumulated in zebrafish to higher concentrations compared to L-selenomethionine. Thus, for the selenium compounds studied, the toxicity appears to at least approximately correlate with the level of bioaccumulation. A summary of selenium content in various larval zebrafish tissues is shown in Fig. 4. While more replicates are needed to increase statistical confidence, our results are in broad agreement with results for other aquatic organisms including microorganisms and brine fly larvae: organic forms were found to be much more bioavailable compared to inorganic forms..$^{28-30}$ The same general trend of greater bioavailability for organic selenium forms has been indicated for humans when compounds are gastro-intestinally ingested. ${ }^{31}$

\section{Selenium preferably accumulates in pigmented tissues}

Our results indicate that selenium concentrates in tissues containing melanin-bearing cells (melanophores) irrespective
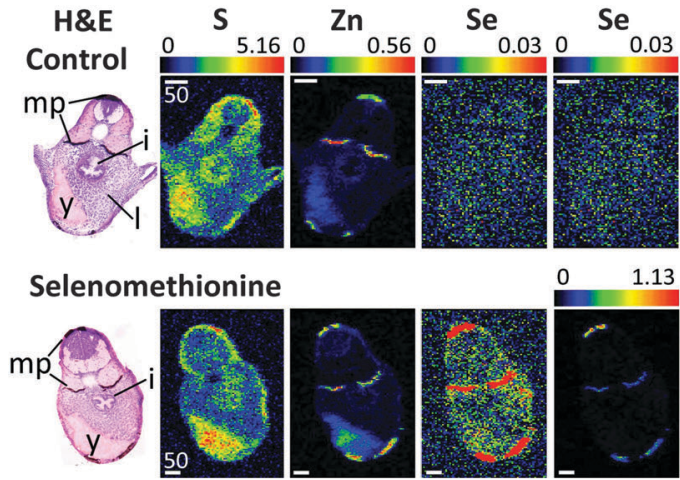

0
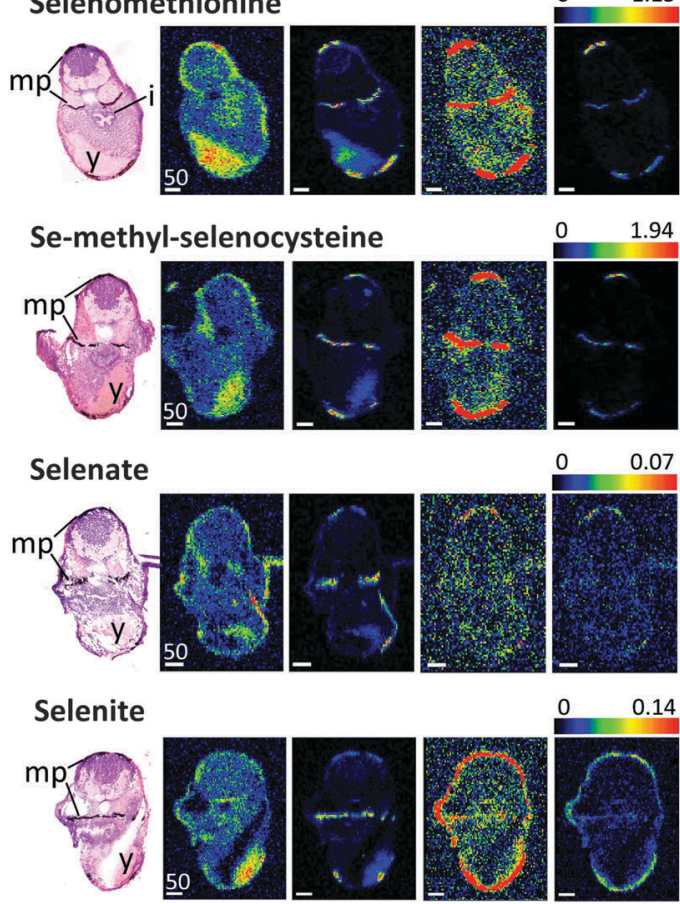

Fig. $3 \mathrm{XFI}$ of trunk sections of larval zebrafish treated with different forms of selenium. Details are similar to those in Fig. 1. Scale bars indicate $50 \mu \mathrm{m}$. Abbreviations: $\mathrm{mp}$ - melanophores, i - intestinal tract, l - liver, y - yolk sac.

of the chemical form administered (Fig. 1-4). In turn, this finding suggests that cellular accumulation into melanophores is very probably occurring. Our previous finding that selenium levels are higher in pigmented areas even in the absence of exogenous selenium suggests that these cells have higher concentrations of selenium uptake transporters, or are able to retain selenium better than other tissues, or both. At present it is unknown whether such selenium accumulation is a function of the developmental stage of fish larvae, or whether adults also retain high selenium concentrations in pigmented tissues. Traditionally, melanin is considered to provide protection against UV-induced oxidative stress and it has been reported that melanophores maintain higher concentrations of reactive oxygen species compared to other epidermal cell types even without any external stress. ${ }^{32}$ Because selenoenzymes are used to counter oxidative damage, ${ }^{33,34}$ it is possible that the presence of high concentrations of selenium in these tissues might be related to higher levels of oxidative stress.

To our knowledge, there are no reports on any specific role of selenium in melanin-containing cells, although there may be some links between human neuromelanin, selenoproteins and Parkinson's disease. ${ }^{35}$ Motor dysfunction in Parkinson's disease is believed to be a result of the death of neuromelanin-containing 


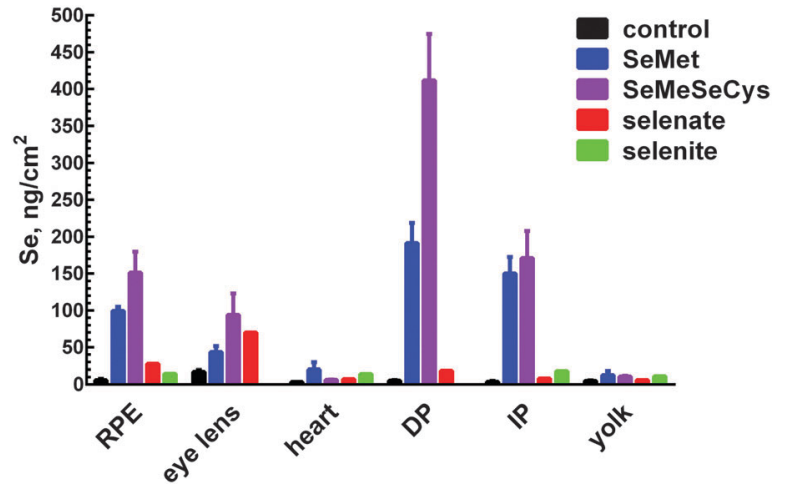

Fig. $4 \mathrm{XFI}$-determined selenium levels from selected tissues of zebrafish larvae, following treatment with different chemical forms. Error bars, where shown, indicate \pm the standard deviation calculated from three replicate sections. RPE indicates the retinal pigmented epithelium, DP the dorsal pigment spot and IP the interior pigment spot. No values for eye lens and DP are given for selenite because the selenite sections examined lacked lenses, and because Se levels in DP were too low to be measured.

dopaminergic neurons in the substantia nigra. It has been reported that selenium dependant glutathione peroxidase 4 is co-localized with neuromelanin, and appears to be involved in regulation of neuromelanin synthesis. ${ }^{35}$

Because both zinc and selenium appear localized with larval zebrafish pigmented tissues ${ }^{13,17}$ we analysed the correlation between these two elements. Fig. 5 shows correlation plots of Se vs. $\mathrm{Zn}$ in the trunk sections of $\mathrm{L}$-selenomethionine and Se-methyl-L-selenocysteine treated zebrafish. The correlation plots have two distinctive domains, one of which corresponds to the dorsal pigment spot showing a higher Se/Zn ratio (purple), and the other to both the internal and the ventral pigment spot, with a lower $\mathrm{Se} / \mathrm{Zn}$ ratio (red). Both domains have high $R^{2}$ values $(\geq 0.85)$ suggesting that zinc and selenium are significantly correlated in both regions, and both organic forms of selenium show similar patterns with Se/Zn molar ratios that are 3.6 and 3.9 fold higher for dorsal than for ventral and internal pigment spots, with L-selenomethionine and Se-methyl-L-selenocysteine, respectively. These Se/Zn molar ratios were remarkably consistent across replicate data sets. Thus, L-selenomethionine gave Se/Zn molar ratios of $2.5 \pm 0.3$ and $0.63 \pm 0.06$ (mean \pm standard deviation) for dorsal and internal/ventral pigment spots, respectively. For Se-methyl-L-selenocysteine the corresponding values are $4.5 \pm 0.1$ and $1.25 \pm 0.07$. Accumulation of selenium in these regions for inorganic forms was too low to determine whether similar correlations are present. This differential accumulation depending on location might be due to maturity of melanophores; dorsal melanophores are expected to be more mature relative to internal and ventral melanophores, or to other unknown differences between the cells in the pigment spots, such as differential expression of selenium transporters.

Interestingly, MacDonald et al. showed that treatment of zebrafish larvae with inorganic mercury depletes selenium from pigmented areas of the zebrafish larvae. ${ }^{13}$ The ability of pigmented tissues to accumulate large concentrations of selenium and then to use them to bind and possibly detoxify mercury
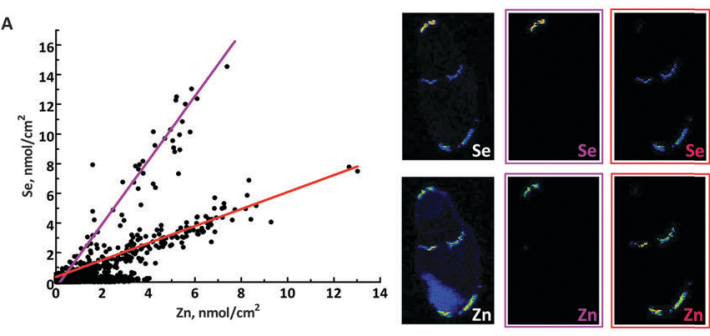

B
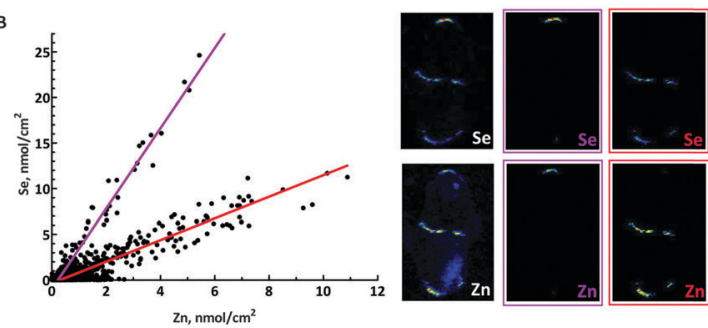

Fig. 5 Se vs. Zn correlation plots from the trunk section XFI of zebrafish larvae treated with (A) L-selenomethionine and (B) Se-methyl-selenocysteine together with corresponding XFI (insets). Plots show two distinct correlation domains: the data with purple outlines correspond to dorsal pigment spots [Se/Zn of 2.2 and 4.4 for SeMet and SeMeSeCys, respectively]. The data with red outlines includes internal and ventral pigment spots [Se/Zn of 0.6 and 1.2 for SeMet and SeMeSeCys, respectively]. The correlation between Se and $\mathrm{Zn}$ in both domains is significant with $R^{2}>0.85$.

suggests that these cells might serve as a reservoir for labile selenium. In recent years, it has become increasingly clear that melanophores and melanocytes have additional functions other than pigment production and associated UV protection. ${ }^{36}$ The relationship with selenium may be an additional example of another function for melanophores.

\section{Accumulation of selenium in the eye sections of zebrafish}

Accumulation of selenium in eye sections of zebrafish larvae treated with different forms of selenium is illustrated in Fig. 1. The highest concentration of selenium in sections from organic selenium treatments is found in melanin-containing RPE cells (Fig. 1 and 4). Selenium is also elevated in eye lens; for organic forms its concentration in this tissue is lower than in pigmented cells. However for the selenate treatment the lens contained the highest selenium levels, comparable with the levels observed with organic forms. Accumulation of selenium in the eye lens was also observed for maternally transferred selenium. ${ }^{14}$ The eye lens is also rich in sulfur, as can also be seen in Fig. 1. Correlation analysis of Se $v s$. S in the eye lens section (Fig. 6) showed that these elements are significantly correlated $\left(R^{2}=0.61-0.83\right)$. Fish eye lens contains more than $1 \mathrm{~g} \mathrm{ml}^{-1}$ of crystallins and $\sim 50 \%$ of this is $\gamma$-crystallins. ${ }^{37} \gamma$-Crystallins are rich in methionine, and we first considered whether the observed Se $v s$. S concentration in eye lens might be due to translational substitution of L-methionine by L-selenomethionine. We previously adopted this explanation for the high maternal selenium transfer observed in our earlier work, ${ }^{14}$ and used micro-X-ray absorption spectroscopy to confirm that the eye lens selenium is indeed present as selenomethionine. ${ }^{14}$

Because lens fibres are added to the growing lens from the lens centre outwards, more mature cells are expected to reside 
A

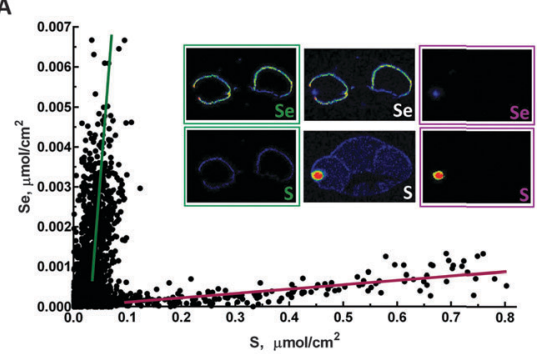

B

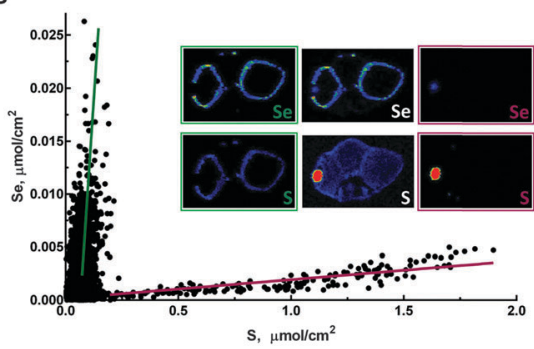

Fig. 6 Correlation plots with corresponding XFI of selenium and sulfur in eye sections of L-selenomethionine- (A) and Se-methyl-L-selenocysteine- (B) treated zebrafish larvae. Coloured borders outline two different domains: green domain include regions with high selenium and low sulfur concentration in RPE and melanophores, while purple domain includes regions with high sulfur and slightly elevated selenium levels in the eye lens.

in the middle of the lens. ${ }^{38}$ Mature lens fibres are anucleate and do not express appreciable amounts of new crystallin proteins. Hence if co-translational incorporation of $\mathrm{L}^{-}$-selenomethionine in place of $\mathrm{L}^{-}$ methionine is responsible for the high lens selenium observed following our treatments of $24 \mathrm{~h}$ starting at $72 \mathrm{hpf}$, higher selenium levels would be expected at the periphery of the eye lens with almost no selenium in the middle, forming a ring outlining the lens in the XFI of our sections. In contrast, our XFI data show that selenium is correlated with sulfur, and has maximum levels in the middle of the lens. Thus, our XFI data indicates that co-translational substitution of L-methionine with L-selenomethionine is not the primary mechanism for lens accumulation in the presence of exogenous selenium. Instead a different mechanism must be operating, allowing selenium to penetrate into the more mature portions of the lens, perhaps an abiotic mechanism associated with the greater hydrophobicity of the lens interior relative to the exterior.

In contrast to the eye lens, accumulation of selenium in melanophore containing tissues in zebrafish eye sections (e.g. the RPE) shows no significant correlation with sulfur $\left(R^{2}=0.08-\right.$ $0.16)$, and most likely represents mechanisms of selenium accumulation that are discrete from those operating in the lens (Fig. 6). Zebrafish and other fish from the class Actinopterygii possess selenoproteins from the SelJ family, which have been found both in eye lens and in neural crest cells. ${ }^{39}$ Such proteins are homologues of J1-crystallins and may play structural or other roles in the developing eye lens. ${ }^{39}$ At present there is no information on the expression level of this protein relative to overall selenium levels, and future studies are needed to understand its relationship with selenium levels in the larval zebrafish eye lens.

Treatment with either organic or inorganic selenium compounds leads to increase of selenium content in melanin-containing
RPE (Fig. 1). Besides the previously discussed functions of all melanophores in preventing oxidative stress, RPE is a major component of the visual cycle responsible for regeneration of visual pigment, and also is the tissue playing the main role in regeneration of photoreceptor membranes. ${ }^{40}$ While all of these functions are expected to require higher than normal antioxidative defence provided by selenoproteins, we cannot exclude the possibility that selenium plays some unknown specific role in RPE. Thisse et $a .^{41}$ have studied selenoprotein mRNA distribution in zebrafish larvae; mRNAs of several selenoproteins were found in the retina either in the region of the RPE or close to it. These included phospholipid hydroperoxide, glutathione peroxidase 4, thioredoxin reductases 1 and 2, iodothyronine deiodinase 2, selenoproteins $\mathrm{W}, \mathrm{T}$ and $\mathrm{H}$, selenophosphate synthetase 2 and SeP15. ${ }^{41}$ The functions of some of these proteins are still unknown and it is possible that they may play specific roles in melanophore metabolism. The difference between the levels of selenium in RPE with and without treatment is very high, increasing by approximately a factor of 30 with Se-methyl-L-selenocysteine treatment. Such a high increase in selenium content suggests that incorporation of selenium into selenoproteins is not the sole cause of accumulation. Binding of selenium to sulfur containing species such as glutathione can also be excluded as a major cause of selenium accumulation, because selenium and sulfur are not correlated in RPE (Fig. 6).

\section{Selenium accumulation in other tissues}

High levels of selenium can also be seen in the heart region of the treated fish (Fig. 2), regardless of the selenium form given. It is possible that this accumulation reflects high concentrations of selenium in the blood and not in the heart tissue itself: as mentioned earlier, treatment of zebrafish with selenium compounds caused hemoglobin discoloration. It is possible that the toxic effects of selenium compounds may be caused in part by binding of metabolites of selenium compounds to hemoglobin, thus preventing oxygen from reaching tissues. Selenide, which will be [HSe $]^{-}$at physiological $\mathrm{pH}$, plays a central role in selenium metabolism; ${ }^{5}$ changes in the visual spectrum of hemoglobin under the influence of hydrogen selenide were reported as early as $1891,{ }^{42}$ with color changes from red to olive-green upon chalcogenide binding. ${ }^{42}$ Similar color changes have been observed in human blood and are attributed to chalcogenide binding to heme iron. ${ }^{43}$ Fig. S5 (ESI $\dagger$ ) shows an example of iron and selenium elemental maps of the heart region of a selenomethionine-treated fish. Both iron and selenium levels are elevated in the heart, with a significant correlation between the two elements $\left(R^{2}=0.44\right)$. It is possible that selenium compounds or their metabolites might additionally interfere with heme synthesis by causing porphyrin or iron deficiencies.

XFI shows that while the level of selenium is slightly higher in the skin layer of the fish larvae after treatment with all tested chemical forms, the treatment with selenite leads to substantial amounts of selenium concentrated in the skin layer or directly below it (Fig. 1-3, last row). One of the possible explanations is that selenite reacts with mucosal polysaccharides or other molecules within the epithelium. It is also possible that selenite is specifically imported into the skin cells, and it would be very interesting to know what transporter is responsible for specific 
uptake of selenite into the cells. Other possibilities are accumulation of selenium in keratinocytes of zebrafish, cells that also contain melanin or accumulation in xanthophores and/or iridophores that produce different types of pigment, but might require protection from oxidative stress.

The yolk sac is another larval organ found to accumulate selenium after treatment with either organic or inorganic forms (Fig. 3). Similar to eye lens, yolk is very rich in sulfur, and the mechanism of selenium accumulation may be related to the mechanism of uptake into the eye lens.

\section{Conclusions}

Treatment of zebrafish larvae with different forms of selenium revealed correlation between toxicity and bioaccumulation: the more toxic organic forms (L-selenomethionine and Se-methyl-Lselenocysteine) appear to accumulate to higher concentrations in the fish larvae compare to the less toxic inorganic forms (selenate and selenite) despite much lower doses for the organic forms. In the organic treatments, selenium preferentially accumulates in the melanin-containing cells, where in some cases selenium concentrations are elevated up to 80 times above endogenous levels. In addition to the pigmented regions, selenium is accumulated in eye lens, yolk, heart, and blood. Future work should include identification of selenium-containing proteins or compounds and speciation of selenium chemical form stored in melanincontaining tissues.

\section{Acknowledgements}

We thank members of the George-Pickering research group and beamline staff at SSRL for their assistance. This work was supported by Natural Sciences and Engineering Research Council of Canada Discovery Grants (to IJP, and to PHK) and by the Saskatchewan Innovation and Science Fund (IJP). GNG and IJP are Canada Research Chairs. ND is supported by the Saskatchewan Health Research Foundation GEMS Research Group Grant (Nichol, Group leader) and is a CIHR-THRUST Associate. MJH, TCM, SN and AKJ are CIHR-THRUST Fellows; MJH is supported by a CIHR Post-Doctoral Fellowship award and by the CIHR/HSFC Team in Synchrotron Medical Imaging. Use of the Stanford Synchrotron Radiation Lightsource (SSRL), SLAC National Accelerator Laboratory, is supported by the U.S. DOE, Office of Science, OBES under Contract No. DE-AC02-76SF00515. The SSRL Structural Molecular Biology Program is supported by the DOE Office of Biological and Environmental Research, and by the National Institutes of Health (NIH), National Institute of General Medical Sciences (NIGMS) (including P41GM103393). The contents of this publication are solely the responsibility of the authors and do not necessarily represent the official views of NIGMS or NIH.

\section{References}

1 V. M. Labunskyy, D. L. Hatfield and V. N. Gladyshev, Selenoproteins: Molecular pathways and physiological roles, Physiol. Rev., 2014, 94, 739-777.
2 A. J. Duffield-Lillico, M. E. Reid, B. W. Turnbull, G. F. Combs Jr., E. H. Slate, L. A. Fischbach, J. R. Marshall and L. C. Clark, Baseline characteristics and the effect of selenium supplementation on cancer incidence in a randomized clinical trial: A summary report of the nutritional prevention of cancer trial, Cancer Epidemiol., Biomarkers Prev., 2002, 11, 630-639.

3 D. L. Hatfield and V. N. Gladyshev, The outcome of selenium and vitamin E cancer prevention trial (SELECT) reveals the need for better understanding of selenium biology, Mol. Interventions, 2009, 9, 18-20.

4 M. Vinceti, C. M. Crespi, C. Malagoli, C. Del Giovani and V. Krogh, Friend or foe? The current epidemiological evidence on selenium and human cancer risk, J. Environ. Sci. Health, Part C: Environ. Carcinog. Ecotoxicol. Rev., 2013, 31, 305-341.

5 C. M. Weekley and H. H. Harris, Which form is that? The importance of selenium speciation and metabolism in the prevention and treatment of disease, Chem. Soc. Rev., 2013, 42, 8870-8894.

6 S. Schlegel and P. Gut, Metabolic insights from zebrafish genetics, physiology, and chemical biology, Cell. Mol. Life Sci., 2015, 72, 2249-2260.

7 J. Barriuso, R. Nagaraju and A. Hurlstone, Zebrafish: A new companion for translational research in oncology, Clin. Cancer Res., 2015, 21, 968-975.

8 D. M. Janz, D. K. DeForest, M. L. Brooks, P. M. Chapman, G. Gilron, D. Hoff, W. D. Hopkins, D. O. McIntyre, C. A. Mebane, V. P. Palace, J. P. Skorupa and M. Wayland, Selenium toxicity to aquatic organisms, in Ecological Assessment of Selenium in the Aquatic Environment, ed. P. M. Chapman, W. J. Adams, M. Brooks, C. G. Delos, S. N. Luoma, W. A. Maher, H. M. Ohlendorf, T. S. Presser and D. P. Shaw, CRC Press, Taylor \& Francis, Boca Raton, 2010.

9 J. K. Thomas and D. M. Janz, Dietary selenomethionine exposure in adult zebrafish alters swimming performance, energetics and the physiological stress response, Aquat. Toxicol., 2011, 102, 79-86.

10 J. K. Thomas and D. M. Janz, In ovo exposure to selenomethionine via maternal transfer increases developmental toxicities and impairs swim performance in $\mathrm{F} 1$ generation zebrafish (Danio rerio), Aquat. Toxicol., 2014, 152, 20-29.

11 J. K. Thomas, S. Wiseman, J. P. Giesy and D. M. Janz, Effects of chronic dietary selenomethionine exposure on repeat swimming performance, aerobic metabolism and methionine catabolism in adult zebrafish (Danio rerio), Aquat. Toxicol., 2013, 130-131, 112-122.

12 A. J. Masse, J. K. Thomas and D. M. Janz, Reduced swim performance and aerobic capacity in adult zebrafish exposed to waterborne selenite, Comp. Biochem. Physiol., Part C: Toxicol. Pharmacol., 2013, 157, 266-271.

13 T. C. MacDonald, M. Korbas, A. K. James, N. J. Sylvain, M. J. Hackett, S. Nehzati, P. H. Krone, G. N. George and I. J. Pickering, Interaction of mecrury and selenium in the larval stage zebrafish vertebrate model, Metallomics, 2015, 7, 1247-1255.

14 S. Choudhury, J. K. Thomas, N. J. Sylvain, O. Ponomarenko, R. A. Gordon, S. M. Heald, D. M. Janz, P. H. Krone, I. Coulthard, G. N. George and I. J. Pickering, Selenium preferentially 
accumulates in the eye lens following embryonic exposure: A confocal X-ray fluorescence imaging study, Environ. Sci. Technol., 2015, 49, 2255-2261.

15 M. J. Pushie, I. J. Pickering, M. Korbas, M. J. Hackett and G. N. George, Elemental and chemically specific X-ray fluorescence imaging of biological systems, Chem. Rev., 2014, 114, 8499-8541.

16 M. Korbas, S. R. Blechinger, P. H. Krone, I. J. Pickering and G. N. George, Localizing organomercury uptake and accumulation in zebrafish larvae at the tissue and cellular level, Proc. Natl. Acad. Sci. U. S. A., 2008, 105, 12108-12112.

17 T. C. MacDonald, S. Nehzati, N. J. Sylvain, A. K. James, M. Korbas, S. Caine, I. J. Pickering, G. N. George and P. H. Krone, Phenylthiourea alters toxicity of mercury compounds in zebrafish larvae, J. Inorg. Biochem., 2015, 151, 10-17.

18 M. Korbas, B. Lai, S. Vogt, S. C. Gleber, C. Karunakaran, I. J. Pickering, P. H. Krone and G. N. George, Methylmercury targets photoreceptor outer segments, ACS Chem. Biol., 2013, 8, 2256-2263.

19 M. Korbas, T. C. MacDonald, I. J. Pickering, G. N. George and P. H. Krone, Chemical form matters: differential accumulation of mercury following inorganic and organic mercury exposures in zebrafish larvae, ACS Chem. Biol., 2012, 7, 411-420.

20 M. J. Hackett, J. A. McQuillan, F. El-Assaad, J. B. Aitken, A. Levina, D. D. Cohen, R. Siegele, E. A. Carter, G. E. Grau, N. H. Hunt and P. A. Lay, Chemical alterations to murine brain tissue induced by formalin fixation: implications for biospectroscopic imaging and mapping studies of disease pathogenesis, Analyst, 2011, 136, 2941-2952.

21 A. J. Niimi and A. N. LaHam, Relative toxicity of organic and inorganic compounds of selenium to newly hatched zebrafish (Brachydanio rerio), Can. J. Zool., 1976, 54, 501-509.

22 M. C. Arnold, J. E. Forte, J. S. Osterberg and R. T. Di Giulio, Antioxidant rescue of selenomethionine-induced teratogenesis in zebrafish embryos, Arch. Environ. Contam. Toxicol., 2015, DOI: $10.1007 /$ s00244-015-0235-7.

23 H. J. Thompson, L. D. Meeker and S. Kokoska, Effect of an inorganic and organic form of dietary selenium on the promotional stage of mammary carcinogenesis in the rat, Cancer Res., 1984, 44, 2803-2806.

24 P. A. McAdam and O. A. Levander, Chronic toxicity and retention of dietary selenum fed to rats as D- or L-selenomethionine, selenite, or selenate, Nutr. Res., 1987, 7, 601-610.

25 W. Krittaphol, A. McDowell, C. D. Thomson, M. Mikov and J. P. Fawcett, Biotransformation of L-selenomethionine and selenite in rat gut contents, Biol. Trace Elem. Res., 2011, 139, 188-196.

26 D. A. Newsome and R. J. Rorhma, Zinc uptake in vitro by human retinal pigmented epithelium, Invest. Ophthalmol. Visual Sci., 1987, 28, 1795-1799.

27 D. A. Samuelson, P. Smith, R. J. Ulshafer, D. G. Hendricks, R. D. Whitley, H. Heldricks and N. C. Leone, X-ray microanalysis of ocular melanin in pigs maintained on normal and low zinc diets, Exp. Eye Res., 1993, 56, 63-70.

28 T. N. Rosetta and A. W. Knight, Bioaccumulation of selenate, selenite, and seleno-DL-methionine by the brine fly larvae Ephydra cinerea Jones, Arch. Environ. Contam. Toxicol., 1995, 29, 351-357.
29 J. M. Besser, T. J. Canfield and T. W. La Point, Bioaccumulation of Organic and Inorganic Selenium in a Laboratory Food Chain, Environ. Toxicol. Chem., 1993, 12, 57-72.

30 E. D. Franz, C. I. E. Wiramanaden, D. M. Janz, I. J. Pickering and K. Liber, Selenium bioaccumulation and speciation in Chironomus dilutus exposed to water-borne selenate, selenite, or seleno-DL-methionine, Environ. Toxicol. Chem., 2011, 30, 2292-2299.

31 J. Moreda-Pineiro, A. Moreda-Pineiro and P. Bermejo-Barrera, In vivo and In vitro testing for selenium and selenium compounds bioavailability assessment in foodstuff, Crit. Rev. Food Sci. Nutr., 2015, DOI: 10.1080/10408398.2014.934437.

32 N. C. Jenkins and D. Grossman, Role of melanin in melanocyte dysregulation of reactive oxygen species, BioMed. Res. Int., 2013, 2013, 908797, 3.

33 M. Roman, P. Jitarub and C. Barbante, Selenium biochemistry and its role for human health, Metallomics, 2014, 6, 25-54.

34 E. M. Hanschmann, J. R. Godoy, C. Berndt, C. Hudemann and C. H. Lillig, Thioredoxins, glutaredoxins, and peroxiredoxins - Molecular mechanisms and health significance: from cofactors to oxidants to redox rignalling, Antioxid. Redox Signaling, 2013, 19, 1539-1605.

35 F. P. Bellinger, M. T. Bellinger, L. A. Seale, A. S. Takemoto, A. V. Raman, T. Miki, A. B. Manning-Bog, M. J. Berry, L. R. White and G. W. Ross, Glutathione peroxidase 4 is associated with neuromelanin in substantia nigra and dystrophic axons in putamen of Parkinson's brain, Mol. Neurodegener., 2011, 6, 8.

36 P. M. Plonka, T. Passeron, M. Brenner, D. J. Tobin, S. Shibahara, A. Thomas, A. Slominski, A. L. Kadekaro, D. Hershkovitz, E. Peters, J. J. Nordlund, Z. Abdel-Malek, K. Takeda, R. Paus, J. P. Ortonne, V. J. Hearing and K. U. Schallreuter, What are melanocytes really doing all day long. . .?, Exp. Dermatol., 2009, 18, 799-819.

37 H. Zhao, Y. Chen, L. Rezabkova, Z. Wu, G. Wistow and P. Schuck, Solution properties of $\gamma$-crystallins: Hydration of fish and mammal $\gamma$-crystallins, Protein Sci., 2014, 23, 88-99.

38 K. A. Soules and B. A. Link, Morphogenesis of the anterior segment in the zebrafish eye, BMC Dev. Biol., 2005, 5, 12.

39 S. Castellano, A. V. Lobanov, C. Chapple, S. V. Novoselov, M. Albrecht, D. Hua, A. Lescure, T. Lengauer, A. Krol, V. N. Gladyshev and R. Guigo, Diversity and functional plasticity of eukaryotic selenoproteins: Identification and characterization of the SelJ family, Proc. Natl. Acad. Sci. U. S. A., 2005, 102, 16188-16193.

40 J. R. Sparrrow, D. Hicks and C. P. Hamel, The retinal pigment epithelium in health and disease, Curr. Mol. Med., 2010, 10, 802-823.

41 C. Thisse, A. Degrave, G. V. Kryukov, V. N. Gladyshev, S. ObrechtPflumio, A. Krol, B. Thisse and A. Lescure, Spatial and temporal expression patterns of selenoprotein genes during embyogeneisis in zebrafish, Gene Expression Patterns, 2003, 3, 525-532.

42 A. Bruere, The direct action of hydrogen sulfide, hydrogen selenide and hydrogen telluride on hemoglobin, J. Anat. Physiol., 1891, 26, 62-75.

43 A. M. Flexman, G. Del Vicario and S. K. W. Schwarz, Dark green blood in the operating theatre, Lancet, 2007, 369, 1972. 